\title{
Nasopalatine Duct Cyst
}

\author{
Brenda L. Nelson • Ronald L. Linfesty
}

Received: 5 February 2010/Accepted: 10 February 2010/Published online: 4 March 2010

(C) US Government 2010

\begin{abstract}
A case of a nasoplatine duct cyst in a 41-yearold male is reviewed. The typical radiologic and histologic findings are presented.
\end{abstract}

Keywords Nasoplatine duct cyst · Maxilla .

Developmental cyst

\section{History}

A 41-year old male presented to his dentist for a routine dental exam. Radiographs showed a lesion of the anterior maxilla. The associated teeth tested vital and the patient could not recall any history of pain or swelling in the area.

\section{Radiographic features}

Panographic and periapical radiographs showed a wellcircumscribed radiolucency in the midline of the anterior maxilla. The lesion was apical to the central incisors and appears to abut the mesial surfaces of both associated lateral incisors. The radiolucency has a heart shape (Fig. 1), due to the superimposition of the nasal spine. No root resorption is noted.

Disclaimer: The opinions and assertions expressed herein are those of the author and are not to be construed as official or representing the views of the Department of the Navy or the Department of Defense.

B. L. Nelson $(\bowtie) \cdot R$. L. Linfesty

Department of Anatomic Pathology, Naval Medical Center San Diego, 34800 Bob Wilson Drive, San Diego, CA 92134-5000, USA

e-mail: brenda.nelson@med.navy.mil

\section{Diagnosis}

Histological examination of hematoxylin and eosin stained slides demonstrated a cyst lined by stratified squamous, pseudo stratified and cuboidal epithelium (Fig. 2). This cyst wall is composed of fibrovascular connective tissue with arteries, veins, minor salivary glands and multiple moderate sized nerves (Fig. 3).

\section{Discussion}

Nasopalatine duct cysts (NPDCs), also known as incisive canal cysts, are the most common non-odontogenic cyst of the gnathic bones. The cyst is so common, in fact, that it will affect approximately one out of every one hundred persons [1]. A developmental cyst, the nasopalatine duct cyst is believed to arise from epithelial remnants of the nasopalatine duct, the communication between the nasal cavity and anterior maxilla in the developing fetus. As fetal development continues, this connection gradually narrows as the bones of the anterior palate fuse. The result is the formation of the incisive canals that carry nerves and vessels, as well as epithelial rest from the degenerated nasoplatine ducts.

Nasopalatine duct cysts affect a wide age range, however, most present in the forth through sixth decades of life. There is a slight male predilection. Patients may be asymptomatic, with the lesion being detected on routine radiographs, however, many will present with one or more symptoms. Complaints are often found to be associated with an infection of a previously asymptomatic nasopalatine duct cysts and consist primarily of swelling, drainage, and pain $[2,3]$. The vitality of near by teeth should not be affected; however, it is not uncommon to see evidence of 


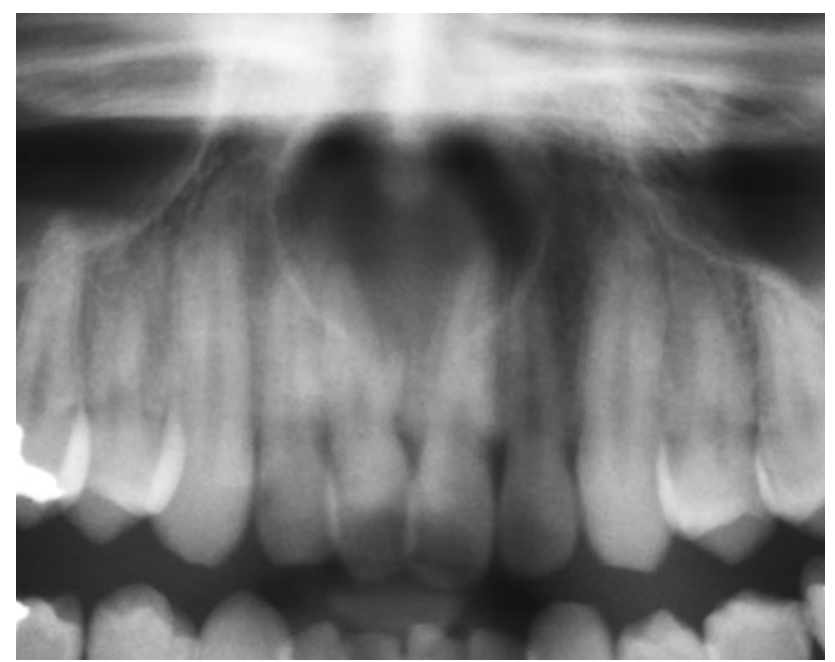

Fig. 1 A large heart shaped radiolucent lesion of the anterior maxilla

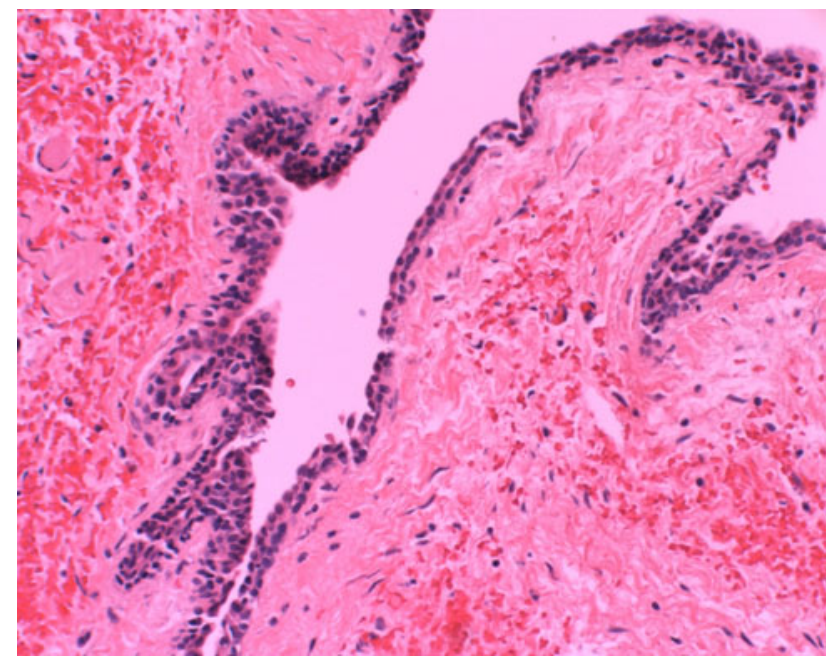

Fig. 2 The nasopalatine duct cyst shows area of flattened cuboidal epithelium

endodontic therapy because the nasopalatine duct cyst was previously clinically misdiagnosed as a periapical cyst or granuloma.

Radiographically, nasopalatine ducts cysts are usually well-circumscribed radiolucencies of the anterior maxilla. The cysts are apical to the roots of the maxillary incisors and rarely cause root resorption. Cysts are round, ovoid or heart shaped due to the superimposition of the nasal spine. Cysts range in size, with an average diameter of approximately $1.5 \mathrm{~cm}$ [1]. The incisive foreman, by convention, is

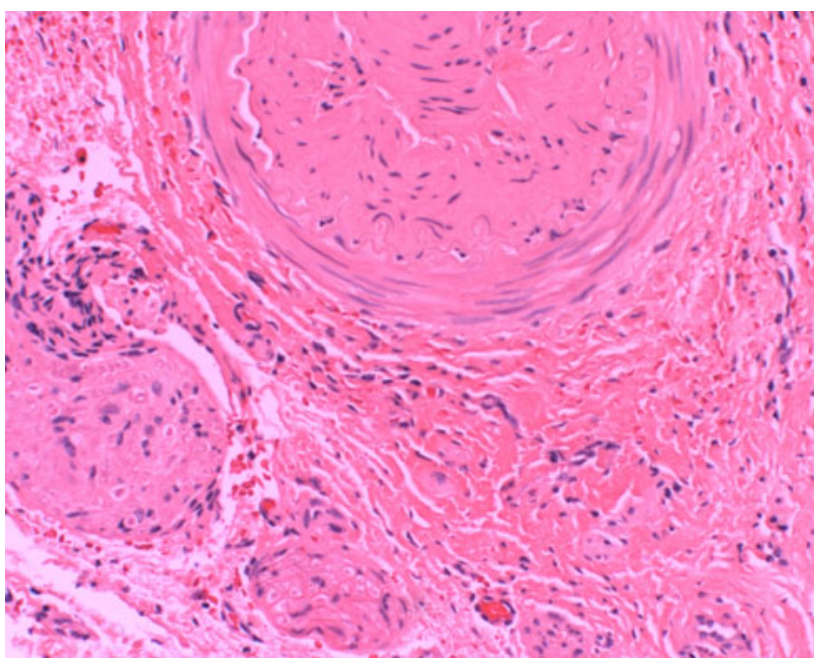

Fig. 3 Areas of large nerves found in the fibrous wall of the cyst

not expected to exceed $6 \mathrm{~mm}$ in diameter, making the detection of a small nasoplatine duct cyst difficult.

Histologically, the nasopalatine duct cyst is lined by stratified squamous epithelium alone or in combination with: pseudostratified columnar epithelium (with or with out cilia and/or goblet cells), simple columnar epithelium, and simple cuboidal epithelium [3]. The fibrous wall generally contains nerves, arteries and veins. Additionally, minor salivary gland tissue and small islands of cartilage may be found. Finally, if the cyst was infected, acute and chronic inflammatory cells will be seen through out the specimen.

Treatment for a nasoplatine duct cyst is complete removal of the lesion, generally by palatal approach. Frequently the biopsy procedure results in adequate treatment. Recurrence is rare [1].

\section{References}

1. Francolí JE, Marqués NA, Aytés LB, Escoda CG. Nasopalatine duct cyst: report of 22 cases and review of the literature. Med Oral Patol Oral Cir Bucal. 2008;13(7):E438-43.

2. Swanson KS, Kaugars GE, Gunsolley JC. Nasopalatine duct cyst: an analysis of 334 cases. J Oral Maxillofacial Surg. 1991;49: 268-71.

3. Vasconcelos R, de Aguiar MF, Castro W, de Araújo VC, Mesquita R. Retrospective analysis of 31 cases of nasopalatine duct cyst. Oral Dis. 1999;5(4):325-8. 